\title{
VEHÍCULOS AUTÓNOMOS: TEMAS VIALES - DILEMAS VITALES. LA TECNOLOGÍA TOMA EL VOLANTE
}

\author{
AUTONOMUS VEHICLES: ROAD ISSUES - VITAL DILEMMAS \\ TECHNOLOGY TAKES THE WHEEL
}

\author{
Ronald Cárdenas Krenz \\ Valeria Padilla Verde ${ }^{* *}$
}

\section{Resumen:}

El artículo constituye una reflexión jurídica con motivo de la aparición de los "vehículos autónomos", los cuales vienen surgiendo bajo la promesa de una alternativa más segura y limpia ante los problemas de inseguridad y caos vehicular, que tanto nos afectan, derivados de la falta de una cultura de cumplimiento respecto a las reglas de tránsito, entre otros factores. Sin embargo, esta nueva tecnología no está exenta de riesgos e implicancias éticas y jurídicas que es preciso advertir antes de que llegue a nuestro medio, perfilándose la necesidad de un marco regulatorio y de control que supervise la aplicación y tecnología de estos vehículos a fin que ofrezcan las garantías necesarias para preservar de la mejor manera no solo los derechos de los usuarios de dichos autos sino también de los peatones.

Palabras Claves: tecnología, vehículos autónomos, ética.

\section{Abstract}

The article is a reflection since the law on the appearance of "autonomous vehicles", which have been emerging under the promise of a safer and cleaner alternative to the problems of insecurity and vehicular chaos, which affect us, derived from the lack of a culture of compliance with traffic rules, among other factors. However, this new technology is not exempt from risks and ethical and legal implications that must be noted before it reaches our environment, outlining the need for a regulatory and control framework that monitors the application and technology of these vehicles in order to offer the necessary guarantees to preserve in the best way not only the rights of the users of these cars but also of the pedestrians.

Doctorando en Derecho. Magíster en Bioética y Biojurídica, y Magíster en Derecho Civil y Comercial. Profesor de las Facultades de Derecho de la Universidad Femenina del Sagrado Corazón, Universidad de Lima y ESAN. Investigador del Instituto de Investigación Científica de la Universidad de Lima. Miembro de la Academia de Derecho y Ciencias Sociales de Córdoba y Miembro de la Pontificia Academia para la Vida.

** Estudiante del décimo ciclo de la Facultad de Derecho de la Universidad de Lima. Asistente de Investigación del Instituto de Investigación Científica de la Universidad de Lima. 
Keywords: technology, autonomous vehicles, ethics.

\section{INTRODUCCIÓN}

Los accidentes de tránsito son hoy en día un problema de salud pública; según la Organización Mundial de la Salud, cada año, más de 1,35 millones de personas mueren en el mundo por culpa de ellos. En el Perú, en el año 2018, conforme cifras del Ministerio de Transportes y Comunicaciones, 3,245 personas murieron en dichas circunstancias; a su vez, otros estudios hablan de cifras que van desde las 3900 hasta las 4300 víctimas mortales al año. ${ }^{23}$ En cualquier caso, los números son alarmantes y la coincidencia unánime es que la principal causa es el exceso de velocidad y la imprudencia de los conductores.

Como dato adicional es de mencionar que, según un estudio de Virginia Tech, el ser humano provoca 4,2 accidentes cada $1600000 \mathrm{~km}$ frente a los 3,2 que provocarían este nuevo tipo de vehículos (Frank et al., 2018); por otro lado, según cifras que cita Salas, el 90\% de los accidentes actuales se deberían a errores o negligencia humana; con lo que, a través de la inteligencia artificial podríamos tener una mayor eficiencia en el tráfico, opciones más baratas para el trasporte y mayor seguridad en las calles (Rouhiainen, 2018, p. 41).

El tema es complejo, sin duda, y no es propósito de estas líneas abordarlo más en detalle, pero sí nos sirve de pretexto para preguntarnos acerca de una nueva tecnología que ya empieza a asomarse: Frente a los problemas de nuestro transporte, en un escenario futurista cada vez menos lejano ¿deberíamos promover el uso de los "vehículos autónomos"?

Asistimos a una era en la que los cambios tecnológicos se dan con inusual intensidad y rapidez; en una época en la que antes de llegar a entender los alcances de una nueva tecnología, ya hay otra que la supera, ofreciendo nuevas posibilidades en las más diversas áreas, que van desde la salud hasta el entretenimiento, pasando por la educación, el trabajo, el desarrollo humano, las comunicaciones, etc.

Uno de los campos que no podía ser ajeno a esta serie de cambios e innovaciones es el del transporte, en cuyo contexto vienen empezando a circular en algunos países, todavía en modo de prueba, autos capaces de

23 Según el Instituto Nacional de Estadística e Informática (INEI, 2018), entre los años 2016 - 2018 se ha experimentado un incremento del número de fallecidos en accidentes de tránsito de 2696 a 3244. 
conducirse por sí mismos, sin que un ser humano esté al volante, formando estos vehículos autónomos parte de una nueva revolución industrial.

En 1925, Francis Houdina presentaba al mundo un automóvil controlado por radio, el cual encendía el motor, cambiaba de marcha y hacía sonar la bocina, generando gran sorpresa y admiración entre el público (Matus, 2017, párr. 3). Sin embargo, esta presentación no estuvo libre de contratiempos, pues quien operaba este vehículo a distancia, a pesar de que pudo esquivar algunos autos, no pudo evitar el impacto contra un sedán.

En todo este devenir, las medidas de seguridad se han venido haciendo cada vez más rigurosas, aunque no por ello podemos dejar de pensar en que siempre habrá posibilidades de error; el reto, en todo caso, es velar por su reducción al mínimo, empero, debemos partir de una cuestión previa: ¿los vehículos autónomos son menos riesgosos que los conducidos por humanos?

\section{AVANCES A TODA VELOCIDAD}

Según un estudio de KPMG, Holanda ocupa el primer lugar del ranking internacional como el país más preparado para el arribo de vehículos autónomos, habiendo el gobierno de dicho país aprobado en el 2015 las primeras pruebas de aplicación de esta tecnología en la vía pública. En Singapur, se viene probando un sistema de taxis autónomos desde el año 2016, denominado "NuTonomy" contando ya con un marco legal para la circulación de este tipo de vehículos. En cuanto a Estados Unidos, que no obstante tiene los casos más difundidos de aplicación, ocupa el tercer lugar en el ranking, en 33 de sus estados se están realizando pruebas con vehículos autónomos con conductor en las vías públicas, siendo dicho país uno de los primeros en registrar un accidente mortal, en el cual un vehículo autónomo atropelló a una mujer que cruzaba la calle, provocándole la muerte ("¿Qué países están más preparados para el coche autónomo sin conductor?", 2018, párr. 3).

Elaine Herzberg, de 49 años de edad, una de las primeras víctimas de los autos autónomos, fue atropellada por uno de estos vehículos de Uber, por un fallo en la programación y configuración del vehículo: el software había estado ajustado erróneamente para ignorar los objetos del camino, viendo a la señora Herzberg como un "falso positivo", por lo que no se desvió antes del accidente. Siendo que la sensibilidad del coche para estos efectos estaba al mínimo, si el conductor humano hubiera estado atento al camino, la tragedia 
no se hubiera producido. Por este accidente, la empresa suspendió todas sus pruebas de vehículos autónomos en Estados Unidos y perdió su permiso para hacer pruebas en Arizona (Álvarez, 2018).

El 7 de enero del 2019, en vísperas de la celebración en Las Vegas de la feria tecnológica más importante del mundo, un robot ruso, llamado Promobot, que iba a ser exhibido en ella, se salió del recinto y fue a parar a una playa de estacionamiento en donde fue atropellado por un auto Tesla conducido mediante piloto automático, siendo herido "mortalmente". Se trata del primer caso en la historia en el que un vehículo autónomo atropella a un robot, convirtiéndolo en "víctima".

El hecho, aparentemente anecdótico, sin embargo, es particularmente relevante pues, por un lado, podría pensarse en qué hubiera pasado si el atropellado no hubiera sido Promobot, sino una persona. Por otro lado, el caso plantea nuevos cuestionamientos a los autos autónomos, respecto a los cuales solo unos días antes del accidente había habido manifestaciones de protesta en diversas ciudades de Estados Unidos. Así, a inicios del 2019, diversos medios daban cuenta que en varias localidades de dicho país, los vecinos atacaron varios autos autónomos de Waymo, que venían siendo objeto de prueba en sus calles. Cabe añadir que, en Tempe, Arizona, en el año 2018, un coche autónomo marca Volvo, de Uber, había golpeado y matado a un peatón.

\section{CONCEPTO. ¿QUÉ SON LOS VEHÍCULOS AUTÓNOMOS?}

Se trata de unidades capaces de imitar las capacidades humanas de manejo, apoyándose en plataformas, programas y sensores electrónicos para la toma de decisiones. De esta manera, se remplaza al chofer por la conducción autómata, a la que se le agrega tecnología de alto nivel ( $\mathrm{Li}$, Díaz, Morantes y Dorati, 2018, p. 34). Sus orígenes se remontan a unos 40 años atrás, hasta llegar a la actualidad, con coches de alta tecnología, incluyendo sistemas de detección láser y de detección de movimientos, pequeñas computadoras de enorme potencia, sensores que identifican magnitudes tanto físicas como químicas en señales o pulsos electrónicos, GPS, actuadores que convierten las señales eléctricas desde la unidad de control en acción controlada, etc. (para más detalle, ver Li et al., 2018).

Las ventajas de los vehículos autónomos son diversas, empezando por la seguridad y el hecho de evitar las fallas o descuidos humanos. Sin embargo, un tema para reflexionar es si podemos pensar en la posibilidad de accidentes derivados ahora de fallas en el equipo, toda 
vez que la tecnología puede también tener deficiencias, fallas, errores de programación y otros.

Por otro lado, si bien podría temerse que a futuro estos vehículos dejen sin empleo a choferes, esta tecnología significará también nuevas oportunidades de trabajo para ingenieros y programadores, entre otras carreras. Ello aparte de demandar menos policías en las calles que distraigan sus funciones para dedicarse a controlar el tránsito.

\section{DILEMAS SOBRE RUEDAS}

No obstante lo expuesto, lo más complejo no viene del lado de la tecnología, sino de lo moral. Podemos programar un auto para que respete las señales de tránsito o no vaya a excesiva velocidad, pero ¿cómo programarlo en aquellas situaciones en las que deba "optar" entre una vida y otra?

En España, el diario El País, en su edición del 3 de enero del 2019,, ${ }^{24}$ planteaba un dilema ético de trascendencia jurídica: si una empresa programa un vehículo autónomo, ¿qué instrucciones debe darle en caso del cruce intempestivo de un peatón sin que haya espacio para frenar: atropellar a la persona para salvaguardar la vida de los pasajeros? ¿Girar el auto hacia la pared del costado a fin de no atropellar al peatón, pero poniendo en riesgo la vida de los pasajeros? ¿Y si el otro es un niño sería nuestra respuesta la misma? ¿Debería resolverse el problema en función del número de pasajeros que van en el auto o teniendo en cuenta el número de personas que están en la pista? Ya, anteriormente, en junio del 2016, el problema lo había planteado públicamente la conocida revista Science. ${ }^{25}$

El viejo dilema del tranvía, ideado por Philippa Foot y enriquecido por Judith Jarvis Thomson, con estas nuevas posibilidades tendrá nuevos retos y protagonistas. Y los profesores de ética tendrán la oportunidad de demostrar a sus alumnos que la ética no es solo una aburrida reflexión teórica, pues tendremos que decidir si nos ponemos a transitar por la vida haciendo uso de las pistas del utilitarismo, la ética de la virtud, el contractualismo, la ética kantiana o tantas otras teorías, para orientar nuestras vidas.

Nos encontramos ante la necesidad de una ética digital (Cortina, 2018), teniendo en cuenta que las tecnologías digitales son objetos legítimos de interés ético, estando los valores humanos insertos en su diseño, como

24 https://elpais.com/elpais/2019/01/02/opinion/1546455278_349208.html

25 Para mayor información ver: https:// www.youtube.com/watch?v=QaR8kxjQ2Bs 
señalan Greene, Lauren y Stark (2019 p. 2126) ${ }^{26}$ agregando que si bien las razones precisas de dicho interés pueden variar de organización en organización, existe consenso en que el impacto positivo o negativo de la inteligencia artificial es un asunto de interés universal.

Como dato curioso, es de observar que, en un estudio hecho por la revista Science en Estados Unidos, la mayoría de los encuestados prefirió que estos actos tengan una moral utilitarista (entre que muera una persona o cinco, menos malo es lo primero que lo segundo), "pero la mayoría asegura que no compraría un coche con estos criterios en su algoritmo" (Salas, 2016, s.p.).

A su vez, un estudio hecho por la revista Nature a partir de un video juego, demuestra que (Criado, 2018):

- Entre un humano y un animal, las personas prefieren salvar al primero y por tanto el auto debería optar por atropellar a una mascota antes que a una persona.

- La gente prefiere salvar al mayor número de personas, por lo que antes que matar a dos peatones, si en el auto va una persona, el coche debería irse contra el muro de al lado.

- Entre atropellar a un niño o a un anciano, debería preferirse al primero para que tenga la oportunidad de envejecer.

- Aunque las ancianas están entre las menos salvadas de entre los humanos, los asiáticos tienden a salvarlas más que los occidentales.

- En Europa y EE UU hay una predilección sutil por las personas de complexión atlética sobre los obesos.

- En los países del sur, se prefiere salvar más a las mujeres que a los hombres.

- En las naciones más desiguales, más participantes prefirieron salvar al peatón con aspecto de ejecutivo.

Sea como sea, frente al clásico dilema, ya los alemanes han tenido que adoptar una posición y, así, el Gobierno Alemán aprobó el "Primer Código Ético relacionado con la conducción autónoma", el cual establece en la regla ética número 9 que cualquier distinción en base a las características personales, como la edad, debe ser prohibido (Awad, Dsouza, Kim, Schulz, Henrich, Shariff, Bonnefon y Rahwan, 2018, p. 2).

Todo ello nos conduce a que la evolución tecnológica que se viene produciendo en el ámbito automotriz requiere de un marco jurídico adecuado

26 "Digital technologies are legitimate objects of ethical concern - and human values are embedded in ther design" (Greene, Lauren, Stark, p. 2126). 
para proteger de la mejor manera la vida, la integridad, la libertad, la salud y el bienestar de las personas.

Dentro de los países que ya han decidido ser los primeros en legislar al respecto, en Estados Unidos, Nevada ha sido el primer estado en autorizar la circulación de coches autónomos, después de que el Departamento del Estado de Vehículos a Motor aprobara que transiten por la vía pública, proyectando a futuro otorgar licencias de vehículos autónomos ("Nevada autoriza la circulación de coches sin conductor", 2012, párr. 1). Asimismo, tenemos la Resolución del Parlamento Europeo, de 16 de febrero de 2017, la cual contiene recomendaciones destinadas a la Comisión respecto a las normas de Derecho Civil sobre robótica; en ellas establecen la necesidad urgente de normas que garanticen el desarrollo de los vehículos autónomos y automatizados con el propósito de explotar su potencial económico y obtener beneficios de los efectos positivos de la tecnología (Parlamento Europeo, 2017). Cabe mencionar, además, que Alemania cuenta ya con el primer código ético para la fabricación y uso de los vehículos autónomos, producto del trabajo de una comisión conformada por 14 expertos (juristas, ingenieros, filósofos, teólogos y expertos en protección del consumidor), liderada por el ex juez del Tribunal Constitucional alemán Udo di Fabio, quienes formularon las bases éticas respecto de la responsabilidad en caso de accidentes (Sánchez, 2017).

Por lo tanto, debemos tener presente que no solo debemos prepararnos para la llegada de los vehículos autónomos, en términos tecnológicos, sino que es preciso abordar el tema desde una perspectiva bioética, multidisciplinaria, que congregue a científicos, juristas, ingenieros, sociólogos, psicólogos, filósofos, programadores, entre otros, para enfrentar el desafío de crear un marco normativo adecuado, incluyendo por cierto el tema de la responsabilidad extracontractual ante los daños que puedan ocasionar estos nuevos vehículos y la cuestión filosófico-jurídica, de alcance técnico, de determinar qué tan autónomos deben ser los vehículos autónomos, toda vez que siempre dependerán de las pautas e indicaciones específicas con que los hayamos podido haber programado.

Ante un accidente de tránsito provocado por un vehículo autónomo, ¿a quién deberíamos de responsabilizar?, ¿al fabricante, al que lo programó, al que lo compró o al pasajero? ¿podrán los vehículos llegar a tener personalidad jurídica como ya se ha planteado respecto a los robots? ¿cuál debe ser el criterio a la hora de tener que "programar" el destino de una persona involucrada en un accidente de tránsito en donde tengamos que elegir de antemano si debe darse prioridad a la vida de un hombre, una mujer, un 
niño o un anciano? Podríamos pensar en que se prefiera a quien no tuvo la culpa, pero allí tenemos por lo menos, por un lado, el problema de qué pasaría si el responsable del accidente sea la imprudencia de un niño y, por otro lado, que ya no estaríamos solo legislando sino también sancionando anticipadamente a una persona sin que haya tenido de por medio proceso o derecho a la defensa alguno.

\section{A MANERA DE CONCLUSIÓN}

La pregunta, en realidad, no es si deben o no empezar a circular los vehículos autónomos, sino cuándo lo harán, pues no solo existe un interés comercial y los deseos de una mayor comodidad al no tener que manejar, sino también las ventajas que ofrecen el liberarnos de la imprudencia y una serie de errores humanos.

Sin embargo, como anota Criado (2018), "habrá situaciones en las que un fallo mecánico, el tiempo o las condiciones de la carretera hagan inevitable el accidente. En esos casos las máquinas deberán decidir entre dos males y para ello habrá que dotarlas de unos principios morales". Por ello, es de tener claro que con los vehículos autónomos habrá menos accidentes, pero también nuevas discusiones.

La regulación sobre la materia, todavía es incipiente, pero, en Alemania, el Código propuesto para los conductores establece en su novena norma un buen punto de partida:

In the event of unavoidable accident situations, any distinction based on personal features (age, gender, physical or mental constitution) is strictly prohibited. It is also prohibited to offset victims against one another. General programming to reduce the number of personal injuries may be justifiable. Those parties involved in the generation of mobility risks must not sacrifice non-involved parties. ${ }^{27}$

Como observa con buen criterio Adela Cortina (2018), a pesar de que haya hecho fortuna la expresión "vehículo autónomo", en verdad no lo es. "Es autómata, y no autónomo; otros le han inscrito las pautas a seguir. Y esta distinción es de la mayor trascendencia" El vehículo autónomo no piensa, "actúa" según lo programado.

Entonces, lo fundamental no es tanto pensar en qué deben hacer los coches autónomos cuando se enfrenten a dilemas éticos, sino qué es lo que

\footnotetext{
Germany. Federal Ministry of Transport and Digital Infraestructure. Report. June 2017.
} 
queremos que hagan, pues los valores humanos están insertos en su diseño programacional.

Felizmente, como dice Cortina, "distintos organismos están asumiendo su responsabilidad en este asunto y surgen propuestas de marcos éticos como la Declaración del Grupo de la Comisión Europea sobre Inteligencia Artificial, robótica y sistemas autónomos, los Principios Asilomar de la Inteligencia Artificial o la Declaración de Derechos Humanos para un Entorno Digital" presentada por la Universidad de Deusto el 26.11.18 (Cortina, 2018).

El uso de los vehículos autónomos nos presentará nuevas versiones del "dilema del tranvía", como desafío ético y normativo, a la hora de programar a quién se salva y a quién no. Sin embargo, como refiere un estudio publicado en Science, la gente no quiere que sea el Gobierno quien obligue a los vehículos autónomos a tener ese espíritu utilitarista que le permita escoger la muerte del pasajero (Salas, 2016).

Por todo ello, no debemos esperar a que llegue esta tecnología para recién pensar en cómo regularla. El debate está abierto y debemos apurarnos, antes de que nos agarre el tráfico, a fin de velar porque los derechos humanos puedan transitar por las pistas, teniendo a la Constitución como nuestra principal señal de tránsito.

\section{REFERENCIAS}

Álvarez, R. (07 de mayo de 2018). El coche autónomo de Uber sí habría detectado al peatón durante al accidente fatal, pero "decidió" no detenerse. Xataca. Recuperado de: https:/ /www.xataka.com/

Awad, E., Dsouza, S., Kim, R., Schulz, J., Henrich, J., Shariff, A., Bonnefon, J. y Rahwan, I. (2018). The Moral Machine experiment. Nature, 563, pp.59 - 64. https://doi.org/10.1038/s41586-018-0637-6

Barrio, M. (2018). Derecho de los Robots. Obtenido de: http://www.elibro. com/

Centro Nacional de Epidemología, Prevención y Control de Enfermedades (2018) Factores que originaron los accidentes de tránsito en el Perú. Obtenido de: https: / / www.dge.gob.pe/portal/docs/vigilancia/sa la/2019/SE24/transito.pdf 
Cortina, A. (07 de diciembre de 2018). Ética Digital. El País. Recuperado de: https://elpais.com/elpais/portada_america.html

Frank, M., Roehrig, P. y Pring, B. (2018). Qué haremos cuando las máquinas lo hagan todo. LID Editorial Empresarial.

Green, D; Laurel, A. y Stark, L. (2019). Better, Nicer, Clearer, Fairer: A Critical Assessment of the Movement for Ethical Artificial Intelligence and Machine Learning. Proceedings of the 52nd Hawaii International Conference on System Sciences. Recuperado de: https://scholarspace.manoa. hawaii.edu/bitstream/10125/59651/0211.pdf

Instituto Nacional de Estadística e Informática (2018). Víctimas de accidentes de tránsito fatales. Recuperado de: https://www.inei.gob.pe/ estadisticas/indice-tematico/traffic-accidents/

Kitman, J. (23 de diciembre del 2016) ¿En verdad son necesarios los vehículos autónomos? The New York Times. Recuperado de: https://www. nytimes.com/es/

Li, Y., Díaz, M., Morantes, S. y Dorati, Y. (2018). Vehículos autónomos: Innovación en la logística urbana. Revista de Iniciación Científica, 4(1), pp. 34-39. https:// doi.org/10.33412/rev-ric.v4.1.1864

Matus, D. (2 de noviembre del 2017). La historia de los carros autónomos contada en unos pocos hitos. Recuperado de: https://es.digitaltrends.com/ autos/historia-carros-autonomos/

Ministerio del Interior (2018). Accidentes de tránsito. Recuperado de: https:// observatorio.mininter.gob.pe/proyectos/accidentes-de-tr\%C3\%A1nsito.

Nevada autoriza la circulación de coches sin conductor (8 de mayo del 2012). Recuperado de: https://motor.elpais.com/actualidad/nevadaautoriza-la-circulacion-de-coches-sin-conductor/

Organización Panamericana de la Salud (7 de diciembre del 2018). Nuevo informe de la OMS destaca que los progresos han sido suficientes en abordar la falta de seguridad en las vías de tránsito del mundo. Recuperado de: https:// www.paho.org/per/index.php?option=com_content\&view=article\&i $\mathrm{d}=$ 4210:nuevo-informe-de-la-oms-destaca-que-los-progresos-han-sidoinsuficientes-en-abordar-la-falta-de-seguridad-en-las-vias-de-transitodel-mundo\&Itemid=1062. 
Parlamento Europeo (16 de febrero de 2017) Resolución del Parlamento Europeo, de 16 de febrero de 2017, con recomendaciones destinadas a la Comisión sobre normas de Derecho civil sobre robótica. Recuperado de: https:/ / www.europarl.europa.eu/doceo/document/A-8-2017-0005_ES.html

¿Qué es y cómo funciona un coche autónomo? (10 de agosto del 2015). Recuperado de: https://www.autobild.es/contenido-patrocinado/especialtoyota-que-es-como-funciona-coche-autonomo-262119

¿Qué países están más preparados para el coche autónomo sin conductor? (11 de julio del 2018). Recuperado de: https://www.hibridosyelectricos. com/articulo/actualidad/paises-estan-mas-preparados-cocheautonomo-conductor/20180711114655020434.html

Rouhiainen, Lasse (2018). Inteligencia artificial. 101 cosas que debes saber hoy sobre nuestro futuro. Editorial Planeta.

Rubio, J. (25 de octubre del 2018) El MIT recurre al dilema filosófico del tranvía para saber a quién podrá atropellar un coche autónomo. El País. Recuperado de: https://elpais.com/elpais/portada_america.html

Salas J. (24 de junio de 2016). ¿Comprarías un coche que elegirá matarte para salvar otras vidas? El País. Recuperado de: https://elpais.com/ elpais/portada_america.html

Sánchez, R. (23 de agosto de 2017). Los coches autónomos alemanes priorizarán la vida sobre los daños materiales en los accidentes. ABC. Recuperado de: https://www.abc.es

Fecha de recepción: 10 de octubre de 2019

Fecha de aceptación: 11 de noviembre de 2019 